\title{
Effect of Chronic Nicotine Exposure on Maternal Renal Adaptations in Pregnant Rats: The Possible Role of Nitric Oxide
}

\author{
ALAA H. MUSALLEM, M.Sc.; SALMA E. NASSAR, M.D.; REHAB E. ABO ELGHEIT, M.D. and \\ RAMEZ A. BARHOMA, M.D.
}

The Department of Physiology, Faculty of Medicine, Tanta University

\begin{abstract}
Background: Nicotine exposure during pregnancy continues to be a widespread public health problem, impacting both fetal and postnatal health. Although the deleterious effects of nicotine on fetal development and the newborn have been extensively investigated, few studies have focused on its negative effects on the maternal adaptations to pregnancy especially relevant on renal functions.
\end{abstract}

Aim of Study: To study the effects of chronic nicotine exposure on the maternal renal adaptations during pregnancy and the possible underlying mechanisms.

Material and Methods: Thirty six adult virgin female albino rats, divided into two main groups, each subdivided into three subgroups; all received treatment for four weeks. Group I (virgin): Subdivided into; saline treated: Received daily subcutaneous saline injections, nicotine treated: Received daily nicotine bitartrate $(1 \mathrm{mg} / \mathrm{kg})$ subcutaneous injections, combined with transdermal nicotine patches $(5.2 \mathrm{mg}$ nicotine/day) application, and nicotine and L-arginine treated: Received the same nicotine therapy plus $0.25 \%$ L-arginine added to drinking. Group II (pregnant): Subdivided into; saline treated: Received daily subcutaneous injections of saline ( 2 weeks prior to mating and $1{ }^{\mathrm{st}}$ two weeks of gestation), nicotine treated: Received daily subcutaneous injections of nicotine bitartrate $(1 \mathrm{mg} / \mathrm{kg})$, with transdermal nicotine patches, and nicotine and L-arginine treated): Received same nicotine therapy plus $0.25 \% \mathrm{~L}$-arginine added to drinking water.

Results: Physiological effects of the pregnancy on renal function were significantly improved by L-argini ne. Similarly, nicotine exposure to virgin group produced a significant increase in serum and urinary $\mathrm{Na}^{+}$, significant decrease in renal total protein, nitric oxide with renal oxidative stress compared to their control group. Co-administration of nicotine and L-arginine to virgin group produced a significant decrease in serum $\mathrm{Na}^{+}$, urinary albumin and renal MDA levels, and significant increase in the urinary $\mathrm{Na}^{+}$level, renal total protein, $\mathrm{NO}$ and GSH levels compared to the virgin nicotine group.

Similarly, nicotine administration to pregnant group produced a significant increase in serum and urinary $\mathrm{Na}^{+}$ FENa, urinary albumin levels, together with significant decrease in GFR, UFR and renal total protein, NO and GSH

Correspondence to: Dr. Alaa H. Musallem,

E-Mail: doctormuslimgirl@gmail.com levels. While co-administration of nicotine and L-arginine to pregnant group produced a significant decrease in serum $\mathrm{Na}^{+}$ FENa, urinary albumin levels, with significant increase in the GFR, UFR and renal total protein, NO and GSH levels.

Conclusion: We concluded that chronic nicotine exposure potentially impaired maternal renal adaptation to pregnancy which was improved greatly by L-arginine, implicating NO as crucial player.

Key Words: L-arginine - Glutathione - Malondialdehyde Nicotine - Pregnancy.

\section{Introduction}

PREGNANT mother undergoes significant anatomical and physiological changes in order to accommodate the developing foetus. These changes affect every organ system in the body [1] .

Although the deleterious effects of nicotine on fetal development and the newborn have been extensively investigated, few studies have focused on its negative effects on the maternal adaptations to pregnancy. There are numerous harmful substances found in tobacco smoke. Nicotine is one of these substances that may be acquired through active and passive smoking. In pregnancy, nicotine exposure through cigarette smoking, nicotine replacement therapies or e-cigarette use, continues to be a widespread public health problem, impacting both fetal and postnatal health. It has been shown that nicotine crosses biological membranes and the blood brain barrier easily. The absorbed nicotine is extensively metabolized in the liver to form a wide variety of metabolites including nicotine $\mathrm{N}$ oxide and cotinine. Nicotine acts by binding to and activating the nicotinic acetylcholine receptors, members of a superfamily of transmembrane ligand-gated ion-channel proteins [1,2]

The aim of this study is to evaluate the effects of chronic nicotine exposure on the maternal renal 
adaptations and the possible underlying mechanisms responsible for the nicotine effects.

\section{Material and Methods}

\section{Animals:}

This study was conducted on 36 adult virgin female albino rats (200-250g), aged 12 to 14 weeks from March 2018 to May 2018 in Physiology Department. These rats were individually housed (except during mating) in metabolic cages and kept under controlled environmental conditions, $12 / 12 \mathrm{~h}$ light/dark cycle, at room temperature $\left(23 \pm 2^{\circ} \mathrm{C}\right)$, with free access to standard rat chow and drinking water. The animals were weighed twice weekly during first two weeks, and then daily during third and fourth week to adjust doses of medication. All procedures were done according to the ethical committee.

\section{Chemicals:}

1-L-arginine: $\mathrm{L}$-arginine was obtained from Biodiagnostic Company in the form of powder $0.25 \%$ which was added to water throughout the experimental period [3]

2- Nicotine preparation: Nicotine bitartrate was purchased from (Sigma, St. Louis, MO, USA), in the form of powder. It was dissolved in $0.9 \%$ saline to obtain a $0.2 \mathrm{mg} / \mathrm{ml}$ concentration of nicotine. PH of nicotine salt solution was adjusted to 7.0-7.4 with five M sodium hydroxide. Doses of nicotine and physiological saline $(\mathrm{NaCl}$ $0.9 \%$ ), in all experimental groups, were calculated to match the weight of the pregnant rats every day, resulting in a delivery of $1 \mathrm{mg} / \mathrm{kg} / \mathrm{day}$.

\section{Study design:}

After two weeks of acclimatization, the animals were classified into 2 main groups; each was further subdivided into 3 subgroups (six rats each):

\section{- Group I (virgin rats): Further subdivided into 3} subgroups;

1-Group Ia Saline treated virgin rats (virgin control, VC): Received subcutaneous injections of saline (vehicle) daily for four weeks. Only the BandAid and waterproof tape were placed on the rat's back, throughout the experimental period.

2- Group Ib Nicotine treated virgin rats (VN): Received subcutaneous injections of nicotine bitartrate (one $\mathrm{mg} / \mathrm{kg} / \mathrm{day}$ ) for four weeks, with daily transdermal nicotine patches applied on the back of each rat for continuous release of $5.2 \mathrm{mg}$ nicotine/rat/day.

3- Group Ic Nicotine and L-arginine treated virgin rats (VN/L-arg): Received the same nicotine therapy, like group $\mathrm{Ib}$, plus $0.25 \% \mathrm{~L}$-arginine added to water and taken by oral gavage throughout the experimental period.

- Group II (pregnant rats): Further subdivided into three subgroups:

1- Group IIa Saline treated pregnant rats (pregnant control, PC): Received subcutaneous injections of saline (vehicle) daily for four weeks (2 weeks prior to mating and ${ }^{1}{ }^{\mathrm{st}} 2$ weeks of gestation). Only the Band-Aid and waterproof tape were placed on the rat's backs, throughout the experimental period.

2- Group IIb Nicotine treated pregnant rats (PN): Received subcutaneous injections of nicotine bitartrate $(1 \mathrm{mg} / \mathrm{kg} /$ day $)$ for four weeks two weeks prior to mating and 1 st two weeks of gestation, with four weeks daily application of transdermal nicotine patches on the back of each rat for continuous release of $5.2 \mathrm{mg}$ nicotine/ $\mathrm{rat} /$ day.

3- Group IIc Nicotine and L-arginine treated pregnant rats (PN/L-arg): Received the same nicotine therapy, like group IIb, plus $0.25 \%$ L-arginine added to water and taken by oral gavage throughout the experimental period.

\section{Sample collection and analysis:}

At the end of four weeks experimental period, 24 hours urine collection was performed for all animals using metabolic cages. Then, all rats were weighed, and anesthetized for blood sampling and decapitated while the kidneys were removed and immediately frozen at $-80^{\circ} \mathrm{C}$ for subsequent analysis.

1- Serum $\mathrm{Na}^{+}(\mathrm{mEq} / \mathrm{L})$ was measured by colorimetric method.

2- Serum creatinine ( $\mathrm{SCr} \mathrm{mg} / \mathrm{dl}$ ) was measured by colorimetric method.

3- Urinary $\mathrm{Na}^{+}(\mathrm{ml} \mathrm{Eq} / \mathrm{L})$ was measured by colorimetric method.

4- Urinary albumin $(\mathrm{mg} / \mathrm{dl})$ was measured by colorimetric method.

5- Urine Flow Rate (UFR).

6- Glomerular Filtration Rate (GFR).

7- Fractional excretion of sodium (FENa).

8- Renal nitrite/nitrate production: Nitric oxide (NO) formation was detected by nitrite/nitrate accumulation in the renal homogenates.

9- Renal total protein content was measured in the renal homogenates. 
10- Renal reduced glutathione (GSH).

11- Renal Malondialdehyde (MDA).

\section{Statistical analysis:}

The data were shown as the mean \pm standard deviation. Data from the study were analyzed using by one-way Analysis of Variance (ANOVA) followed by Tukey's test to assess the significance. The $p<0.05$ were considered as statistically significant. Using SPSS for windows (Version 23.0).

\section{Results}

Daily subcutaneous injection of nicotine bitartrate, together with application of transdermal nicotine patches for four weeks induced a significant increase in serum $\mathrm{Na}^{+}$level, by about $18.1 \%$ and $32.4 \%$. Addition of $0.25 \% \mathrm{~L}$-arginine revealed a significant decrease in the latter values, of VN and PN groups, by about $17.2 \%$ and $13 \%$ in comparison to VN/Larg group (Table 1).

Subcutaneous injection of nicotine bitartrate, together with application of transdermal nicotine patches for four weeks resulted in a significant increase in urinary $\mathrm{Na}+$ level, by about $76.7 \%$ and 260. Co-treatment with both nicotine and $0.25 \%$ L-arginine resulted in a non-significant increase in the later value of VN group by about $13.2 \%$ in comparison to VN/Larg group. However, when compared to the former values of $\mathrm{VC}$ and $\mathrm{PC}$ groups, a significant increase in urinary $\mathrm{Na}^{+}$level by about $100 \%$ was shown in VN/Larg group and $\mathrm{PN} /$ Larg group compared to VC group and PC group respectively Figs. $(1,2)$.

Table (1): Serum $\mathrm{Na}^{+}$level (mEq/L), in virgin and pregnant rats treated with nicotine alone or combined with L-arginine.

\begin{tabular}{|c|c|c|c|c|c|c|}
\hline \multirow[t]{2}{*}{ Groups } & \multicolumn{3}{|c|}{ Group I } & \multicolumn{3}{|c|}{ Group II } \\
\hline & $\mathrm{VC}$ & $\mathrm{VN}$ & VN/Larg & $\mathrm{PC}$ & $\mathrm{PN}$ & PN/Larg \\
\hline 1 & 139.0 & 159.0 & 128.0 & 118 & 149 & 138 \\
\hline 2 & 128.70 & 158.70 & 129.70 & 118.2 & 159.7 & 129.7 \\
\hline 3 & 130.0 & 140.0 & 130.0 & 125 & 140 & 130 \\
\hline 4 & 131.0 & 161.0 & 131.0 & 102 & 155 & 132 \\
\hline 5 & 130.67 & 150.67 & 131.67 & 111 & 156.6 & 131.67 \\
\hline 6 & 129.40 & 159.40 & 129.40 & 112 & 148.4 & 129.4 \\
\hline Range & $128.70-139.0$ & $140.0-161.0$ & $128.0-131.67$ & $102.0-125.0$ & $140.0-159.70$ & $129.40-138.0$ \\
\hline Mean & 131.12 & $154.8 \mathbf{a}$ & $128.1^{\mathbf{b}}$ & $114.36 \mathbf{a}$ & $151.45 \mathrm{c}$ & $131.8 \mathbf{c}^{\mathbf{d}}$ \\
\hline$\pm \mathrm{SD}$ & 3.8 & 8.10 & 1.3 & 7.9 & 7.12 & 3.22 \\
\hline$\%$ change & & $+18.1 \%$ vs. Ia & $\begin{array}{l}-17.2 \% \text { vs. Ib } \\
-2 \% \text { vs. Ia }\end{array}$ & $-12.8 \%$ vs. Ia & $+32.4 \%$ vs. IIa & $\begin{array}{l}-13 \% \text { vs. IIb } \\
+15.3 \% \text { vs. IIa }\end{array}$ \\
\hline $\mathrm{F}$ & & & 39. & 48 & & \\
\hline$p$ & & & & $001 *$ & & \\
\hline VC (Ia) & \multicolumn{3}{|c|}{ : Virgin control group. } & \multicolumn{3}{|c|}{$*$ : Denotes statistical significance $(p<0.05)$. } \\
\hline VN (Ib) & \multirow{2}{*}{\multicolumn{3}{|c|}{$\begin{array}{l}\text { : Virgin nicotine group. } \\
\text { Virgin nicotine and L-arginine group. }\end{array}$}} & \multirow{2}{*}{\multicolumn{3}{|c|}{ a: Denotes $p<0.05$ vs. VC group. }} \\
\hline VN/Larg (Ic) & & & & & & \\
\hline PC (IIa) & \multicolumn{3}{|c|}{ Pregnant control group. } & \multicolumn{3}{|c|}{ c: Denotes $p<0.05$ vs. PC group. } \\
\hline PN (IIb) & \multicolumn{3}{|c|}{ : Pregnant nicotine group. } & \multicolumn{3}{|c|}{ d: Denotes $p<0.05$ vs. PN group. } \\
\hline
\end{tabular}

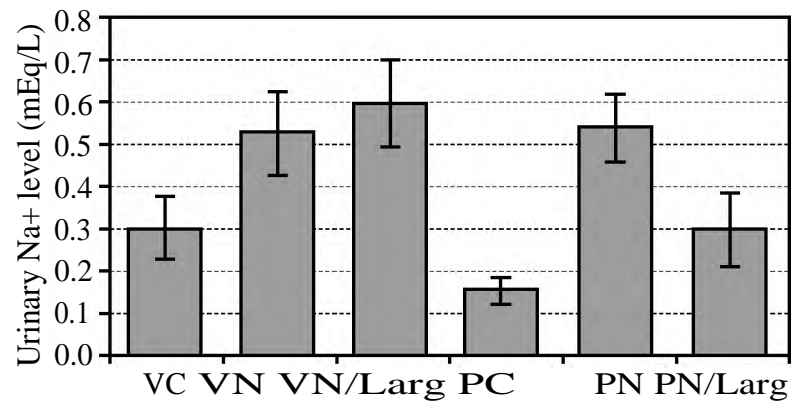

Fig. (1): Urinary $\mathrm{Na}^{+}$level, in virgin and pregnant rats treated with nicotine alone or co mbined with L-arginine.

\footnotetext{
VC (Ia) : Virgin control group.

VN (Ib) : Virgin nicotine group.

VN/Larg (Ic) : Virgin nicotine and L-arginine group.

PC (IIa) : Pregnant control group.

PN (IIb) : Pregnant nicotine group.

PN/Larg (IIc) : Pregnant nicotine and L-arginine group.

a $\quad:$ Denotes $p<0.05$ vs. VC group.

$\begin{array}{ll}\mathrm{c} & : \text { Denotes } p<0.05 \text { vs. PC group. } \\ \mathrm{d} & : \text { Denotes } p<0.05 \text { vs. PN group. }\end{array}$
}

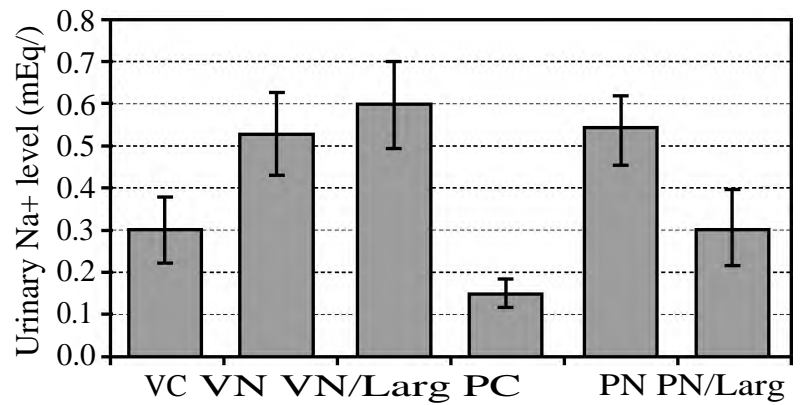

Fig. (2): Percentage change (\%) of urinary $\mathrm{Na}$ level, in all studied groups.

$\begin{array}{ll}\text { VC (Ia) } & \text { : Virgin control group. } \\ \text { VN (Ib) } & \text { : Virgin nicotine group. } \\ \text { VN/Larg (Ic) } & \text { Virgin nicotine and L-arginine group. } \\ \text { PC (IIa) } & \text { : Pregnant control group. } \\ \text { PN (IIb) } & \text { : Pregnant nicotine group. } \\ \text { PN/Larg (IIc) } & : \text { Pregnant nicotine and L-arginine group. } \\ \text { a } & : \text { Denotes } p<0.05 \text { vs. VC group. } \\ \text { c } & : \text { Denotes } p<0.05 \text { vs. PC group. } \\ \text { d } & : \text { Denotes } p<0.05 \text { vs. PN group. }\end{array}$


The four weeks combined nicotine exposure induced a non-significant increase in urinary albumin level, by about $19.4 \%$ and a significant increase in urinary albumin level, by about $40.9 \%$. Addition of $0.25 \%$ L-arginine to water taken by oral gavage throughout the experimental period revealed a significant decrease in the latter values, of $\mathrm{VN}$ and PN groups, by about $30.8 \%$ and $27.1 \%$ in comparison to VN/Larg group (Table 2).

Daily subcutaneous injection of nicotine bitartrate, together with application of transdermal nicotine patches for four weeks induced a nonsignificant decrease in GFR level, by about $20.9 \%$ and a significant decrease in GFR level, by about
$84.5 \%$. Co-treatment with both nicotine and $0.25 \%$ $\mathrm{L}$-arginine resulted in an increase in the GFR level of VN and PN groups by about $29.9 \%$ and $254.8 \%$, in comparison to VN/Larg group (a non-significant increase), and PN/Larg group (a significant increase), respectively (Table 3 ).

Addition of $0.25 \% \mathrm{~L}$-arginine to water taken by oral gavage throughout the experimental period revealed non-significant change in the previous value of fractional sodium excretion in $\mathrm{VN}$ and a significant decrease in the value, of PN group, by about $0 \%$ and $28.3 \%$, respectively in comparison to VN/Larg group (Table 4).

Table (2): Urinary albumin level (mg/dl), in virgin and pregnant rats treated with nicotine alone or combined with L-arginine.

\begin{tabular}{|c|c|c|c|c|c|c|}
\hline \multirow[b]{2}{*}{ No } & \multicolumn{3}{|c|}{ Group I } & \multicolumn{3}{|c|}{ Group II } \\
\hline & $\mathrm{VC}$ & $\mathrm{VN}$ & VN/Larg & $\mathrm{PC}$ & $\mathrm{PN}$ & PN/Larg \\
\hline 1 & 1.5 & 1.8 & 1.2 & 1.5 & 1.8 & 1.5 \\
\hline 2 & 1.6 & 1.4 & 1.5 & 1.21 & 2.6 & 1.4 \\
\hline 3 & 1.3 & 1.5 & 1.3 & 1.5 & 2.3 & 1.3 \\
\hline 4 & 1.1 & 1.71 & 1.1 & 1.6 & 2.1 & 1.2 \\
\hline 5 & 1.8 & 2.2 & 1.4 & 1.8 & 1.8 & 1.8 \\
\hline 6 & 2 & 2.5 & 1.2 & 1.3 & 2 & 2 \\
\hline Range & $1.10-2.0$ & $1.40-2.50$ & $1.10-1.50$ & $1.21-1.80$ & $1.80-2.60$ & $1.20-2.0$ \\
\hline Mean & 1.55 & 1.85 & $1.28 b$ & 1.49 & $2.10 \mathrm{c}$ & $1.53 \mathrm{~d}$ \\
\hline$\pm \mathrm{SD}$ & 0.33 & 0.42 & 0.15 & 0.21 & 0.31 & 0.31 \\
\hline$\%$ change & & $+19.4 \%$ vs. Ia & $\begin{array}{l}-30.8 \% \text { vs. Ib } \\
-17.4 \% \text { vs. Ia }\end{array}$ & $-3.9 \%$ vs. Ia & $+40.9 \%$ vs. IIa & $\begin{array}{l}-27.1 \% \text { vs. IIb } \\
+2.7 \% \text { vs. IIa }\end{array}$ \\
\hline $\mathrm{F}$ & \multirow{2}{*}{\multicolumn{6}{|c|}{$\begin{array}{l}5.66 \\
<0.001 *\end{array}$}} \\
\hline$p$ & & & & & & \\
\hline VC (Ia) & \multicolumn{3}{|c|}{ Virgin control group. } & \multicolumn{3}{|c|}{ PN/Larg (IIc) : Pregnant nicotine and L-arginine group. } \\
\hline VN (Ib) & Virgin nicot & e group. & & & otes statistical sign & ficance $(p<0.05)$ \\
\hline VN/Larg (Ic) & \multicolumn{3}{|c|}{ Virgin nicotine and L-arginine group. } & \multicolumn{3}{|c|}{ es $p<0.05$ vs. VN group. } \\
\hline PC (IIa) & \multicolumn{3}{|c|}{ Pregnant control group. } & \multirow{2}{*}{\multicolumn{2}{|c|}{$\begin{array}{l}\text { es } p<0.05 \text { vs. PC } \\
\text { es } p<0.05 \text { vs. PN }\end{array}$}} & group. \\
\hline $\mathrm{PN}(\mathrm{IIb})$ & Pregnant nic & tine group. & & & & group. \\
\hline
\end{tabular}

Table (3): Glomerular filtration rate level (GFR, $\mathrm{ml} / \mathrm{min}$ ), in virgin and pregnant rats treated with nicotine alone or combined with L-arginine.

\begin{tabular}{|c|c|c|c|c|c|c|}
\hline \multirow[b]{2}{*}{ No } & \multicolumn{3}{|c|}{ Group I } & \multicolumn{3}{|c|}{ Group II } \\
\hline & $\mathrm{VC}$ & VN & VN/Larg & $\mathrm{PC}$ & $\mathrm{PN}$ & PN/Larg \\
\hline 1 & 0.9 & 0.5 & 0.9 & 1.9 & 0.6 & 0.9 \\
\hline 2 & 1.1 & 1 & 1.2 & 2.1 & 0.06 & 1.1 \\
\hline 3 & 1.2 & 0.87 & 1.3 & 2.2 & 0.2 & 1.2 \\
\hline 4 & 1.15 & 0.76 & 1.05 & 2.15 & 0.15 & 1.15 \\
\hline 5 & 1.17 & 1.05 & 1.17 & 1.97 & 0.17 & 1.17 \\
\hline 6 & 1.05 & 1.02 & 1.15 & 2.05 & 0.65 & 1.05 \\
\hline Range & $0.90-1.20$ & $0.50-1.05$ & $0.90-1.30$ & $1.90-2.20$ & $0.06-0.65$ & $0.90-1.20$ \\
\hline Mean & 1.10 & 0.87 & 1.13 & $2.0 \mathrm{a}$ & $0.31^{\mathrm{c}}$ & $1.10^{\mathrm{c}} \mathrm{d}$ \\
\hline$\pm \mathrm{SD}$ & 0.11 & 0.21 & 0.14 & 0.11 & 0.25 & 0.11 \\
\hline$\%$ change & & $-20.9 \%$ vs. Ia & $\begin{array}{l}+29.9 \% \text { vs. Ib } \\
+2.7 \% \text { vs. Ia }\end{array}$ & $+81.8 \%$ vs. Ia & $-84.5 \%$ vs. IIa & $\begin{array}{l}+254.8 \% \text { vs. IIb } \\
-45 \% \text { vs. IIa }\end{array}$ \\
\hline $\mathrm{F}$ & & & & 70.95 & & \\
\hline$p$ & & & & $<0.001 *$ & & \\
\hline VC (Ia) & \multicolumn{3}{|c|}{ Virgin control group. } & \multicolumn{3}{|c|}{ PN/Larg (IIc) : Pregnant nicotine and L-arginine group. } \\
\hline VN (Ib) & \multirow{2}{*}{\multicolumn{3}{|c|}{$\begin{array}{l}\text { Virgin nicotine group. } \\
\text { Virgin nicotine and L-arginine group. }\end{array}$}} & \multicolumn{3}{|c|}{ * $\quad:$ Denotes statistical significance $(p<0.05)$} \\
\hline VN/Larg (Ic) & & & & \multicolumn{3}{|c|}{ Denotes $p<0.05$ vs. VC group. } \\
\hline PC (IIa) & & \multicolumn{3}{|c|}{ : Denotes $p<0.05$ vs. PC group. } \\
\hline PN (IIb) & & & Pregnant nicotine group. & \multicolumn{3}{|c|}{ : Denotes $p<0.05$ vs. PN group. } \\
\hline
\end{tabular}


Table (4): Fractional sodium excretion (FENa\%), in virgin and pregnant rats treated with nicotine alone or combined with L-arginine.

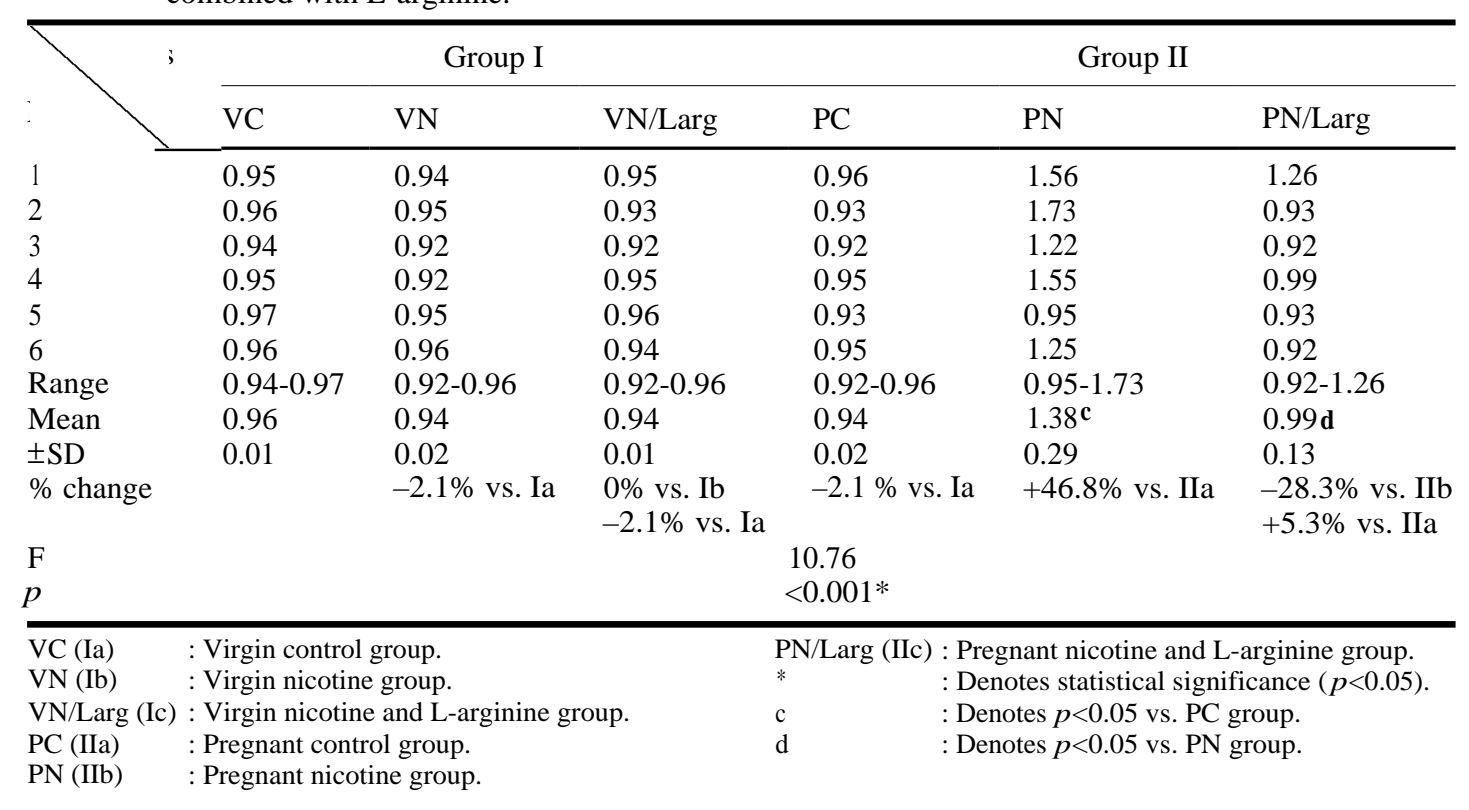

Daily subcutaneous injection of nicotine bitartrate, together with application of transdermal nicotine patches for four weeks induced a nonsignificant decrease in UFR, by about 44.8 and a significant decrease in UFR, by about $59.7 \%$. Addition of $0.25 \% \mathrm{~L}$-arginine to water taken by oral gavage throughout the experimental period revealed a significant increase in the UFR values of VN group, by about $75 \%$ in comparison to VN/Larg group. But, when compared to the former values of $\mathrm{VC}$ and $\mathrm{PC}$ groups, there was a significant decrease in PN/Larg group by about $42.3 \%$ (Table 5).
Daily subcutaneous injection of nicotine bitartrate, together with application of transdermal nicotine patches for four weeks induced a significant decrease in renal NO level, by about $40.6 \%$ and $52.2 \%$. Addition of $0.25 \% \mathrm{~L}$-arginine to water revealed a significant increase in the latter values, of VN and PN groups, by about $104.8 \%$ and $68.8 \%$ in comparison to VN/Larg group. But, when compared to the former values of $\mathrm{VC}$ and $\mathrm{PC}$ groups, it showed a significant increase in renal $\mathrm{NO}$ level of VN/Larg group by about $21.6 \%$ and a significant decrease of PN/Larg group by about $19.4 \%$ (Table 6).

Table (5): Urinary flow rate (UFR, $\mathrm{ml} / \mathrm{min}$ ), in virgin and pregnant rats treated with nicotine alone or combined with L-arginine.

\begin{tabular}{|c|c|c|c|c|c|c|}
\hline \multirow[t]{2}{*}{ Groups } & \multicolumn{3}{|c|}{ Group I } & \multicolumn{3}{|c|}{ Group II } \\
\hline & $\mathrm{VC}$ & VN & VN/Larg & $\mathrm{PC}$ & $\mathrm{PN}$ & PN/Larg \\
\hline 11 & 0.6 & 0.4 & 0.62 & 1.6 & 0.5 & 0.6 \\
\hline 2 & 1.5 & 0.55 & 1.3 & 1.8 & 0.58 & 1.3 \\
\hline 3 & 1.2 & 0.82 & 1.1 & 1.7 & 1.1 & 1.2 \\
\hline 4 & 1.33 & 0.73 & 1.33 & 2.33 & 0.93 & 1.23 \\
\hline 5 & 1.45 & 0.45 & 1.45 & 2.45 & 1.05 & 1.55 \\
\hline 6 & 0.9 & 0.9 & 0.91 & 1.9 & 0.59 & 0.9 \\
\hline Range & $0.60-1.50$ & $0.40-0.90$ & $0.62-1.45$ & $1.60-2.45$ & $0.50-1.10$ & $0.60-1.55$ \\
\hline Mean & 1.16 & 0.64 & $1.12 \mathbf{b}$ & $1.96 \mathbf{a}$ & $0.79 \mathrm{c}$ & $1.13^{\mathrm{c}}$ \\
\hline$\pm \mathrm{SD}$ & 0.35 & 0.20 & 0.31 & 0.35 & 0.27 & 0.33 \\
\hline$\%$ change & & $-44.8 \%$ vs. Ia & $\begin{array}{l}+75 \% \text { vs. Ib } \\
-3.4 \% \text { vs. Ia }\end{array}$ & $+69 \%$ vs. Ia & $-59.7 \%$ vs. IIa & $\begin{array}{l}+43.04 \% \text { vs. IIb } \\
-42.3 \% \text { vs. IIa }\end{array}$ \\
\hline $\mathrm{F}$ & & & & 13.43 & & \\
\hline$p$ & & & & $<0.001 *$ & & \\
\hline VC (Ia) & \multicolumn{3}{|c|}{ Virgin control group. } & \multicolumn{3}{|c|}{ PN/Larg (IIc) : Pregnant nicotine and L-arginine group. } \\
\hline $\mathrm{VN}(\mathrm{Ib})$ & \multirow{2}{*}{\multicolumn{3}{|c|}{$\begin{array}{l}\text { Virgin nicotine group. } \\
\text { Virgin nicotine and L-arginine group. }\end{array}$}} & \\
\hline VN/Larg (Ic) & & & & \multirow{2}{*}{\multicolumn{2}{|c|}{ tes $p<0.05$ vs. }} & group. \\
\hline PC (IIa) & \multicolumn{3}{|c|}{ Pregnant control group. } & & & $\mathrm{N}$ group. \\
\hline $\mathrm{PN}(\mathrm{IIb})$ & \multicolumn{3}{|c|}{ Pregnant nicotine group. } & \multicolumn{2}{|r|}{$p<0.05$ vs. $\mathrm{P}$} & group. \\
\hline
\end{tabular}


Table (6): Renal nitrate/nitrite level (NO, nmol/gm), in virgin and pregnant rats treated with nicotine alone or combined with L-arginine.

\begin{tabular}{|c|c|c|c|c|c|c|}
\hline $\mathrm{s}$ & \multicolumn{3}{|c|}{ Group I } & \multicolumn{3}{|c|}{ Group II } \\
\hline - & $\mathrm{VC}$ & VN & VN/Larg & $\mathrm{PC}$ & $\mathrm{PN}$ & PN/Larg \\
\hline 1 & 500 & 300 & 610 & 650 & 300 & 500 \\
\hline 2 & 450 & 250 & 460 & 460 & 250 & 450 \\
\hline 3 & 512 & 312 & 615 & 625 & 312 & 502 \\
\hline 4 & 478 & 288 & 588 & 598 & 278 & 468 \\
\hline 5 & 489 & 289 & 599 & 599 & 289 & 499 \\
\hline 6 & 501 & 301 & 691 & 691 & 301 & 501 \\
\hline Range & $450.0-512.0$ & $250.0-312.0$ & $460.0-691.0$ & $460.0-691.0$ & $250.0-312.0$ & $450.0-502.0$ \\
\hline Mean & 488.33 & 290.0a & $593.83 \mathbf{a}^{\mathbf{a}} \mathbf{b}$ & $603.83 \mathbf{a}$ & 288.33 c & $486.67 \mathbf{c}^{\prime} \mathbf{d}$ \\
\hline$\pm \mathrm{SD}$ & 22.06 & 21.49 & 75.0 & 78.64 & 22.06 & 22.20 \\
\hline$\%$ change & & $-40.6 \%$ vs. Ia & $\begin{array}{l}+104.8 \% \text { vs. Ib } \\
+21.6 \% \text { vs. Ia }\end{array}$ & $+23.7 \%$ vs. Ia & $-52.2 \%$ vs. IIa & $\begin{array}{l}+68.8 \% \text { vs. IIb } \\
-19.4 \% \text { vs. IIa }\end{array}$ \\
\hline $\mathrm{F}$ & \multirow{2}{*}{\multicolumn{6}{|c|}{$\begin{array}{l}51.61 \\
<0.001 *\end{array}$}} \\
\hline$p$ & & & & & & \\
\hline VC (Ia) & \multicolumn{6}{|c|}{ tatistical significance $(p<0.05)$. } \\
\hline VN (Ib) & \multicolumn{6}{|c|}{ Virgin nicotine group. } \\
\hline VN/Larg (Ic) & \multicolumn{3}{|c|}{ Virgin nicotine and L-arginine group. } & \multicolumn{3}{|c|}{ b : Denotes $p<0.05$ vs. VN group. } \\
\hline $\mathrm{PC}$ (IIa) & \multirow{2}{*}{\multicolumn{3}{|c|}{$\begin{array}{l}\text { Pregnant control group. } \\
\text { Pregnant nicotine group. }\end{array}$}} & \multirow{2}{*}{\multicolumn{3}{|c|}{$\begin{array}{l}\text { c : Denotes } p<0.05 \text { vs. PC group. } \\
d: \text { Denotes } p<0.05 \text { vs. PN group. }\end{array}$}} \\
\hline PN (IIb) & & & & & & \\
\hline PN/Larg (IIc) & \multicolumn{6}{|c|}{ Pregnant nicotine and L-arginine group. } \\
\hline
\end{tabular}

Daily subcutaneous injection of nicotine bitartrate, together with application of transdermal nicotine patches for four weeks induced a significant decrease in renal total protein content, by about $19.2 \%$ and $24.9 \%$. Addition of $0.25 \% \mathrm{~L}$ - arginine to water taken by oral gavage throughout the experimental period revealed a significant increase in the latter values, of VN and PN groups, by about $24.5 \%$ and $34.9 \%$ in comparison to VN/Larg group (Table 7).

Table (7): Renal total protein content level ( $\mathrm{mg} / \mathrm{gm})$, in virgin and pregnant rats treated with nicotine alone or combined with L-arginine.

\begin{tabular}{|c|c|c|c|c|c|c|}
\hline \multirow{2}{*}{$\begin{array}{l}\text { Groups } \\
\text { No }\end{array}$} & \multicolumn{3}{|c|}{ Group I } & \multicolumn{3}{|c|}{ Group II } \\
\hline & $\mathrm{VC}$ & $\mathrm{VN}$ & VN/Larg & $\mathrm{PC}$ & $\mathrm{PN}$ & PN/Larg \\
\hline 1 & 154 & 115 & 144 & 155 & 113 & 164 \\
\hline 2 & 145.6 & 125.6 & 165.6 & 155.6 & 122.6 & 155.6 \\
\hline 3 & 167.5 & 137.5 & 157.5 & 167.5 & 127.5 & 167.5 \\
\hline 4 & 176.5 & 126.5 & 176.5 & 176.5 & 116.5 & 176.5 \\
\hline 5 & 150.7 & 130.7 & 156.7 & 152.7 & 120.7 & 156.7 \\
\hline 6 & 156 & 133 & 156 & 156 & 123 & 156 \\
\hline Range & $145.60-176.50$ & $115.0-137.50$ & $144.0-176.50$ & $152.70-176.50$ & $113.0-127.50$ & $155.60-176.50$ \\
\hline Mean & 158.38 & $128.05 \mathbf{a}$ & $159.38 \mathbf{b}$ & 160.55 & $120.55 \mathrm{c}$ & $162.72^{d}$ \\
\hline$\pm \mathrm{SD}$ & 11.48 & 7.74 & 10.87 & 9.38 & 5.14 & 8.32 \\
\hline$\%$ change & & $-19.2 \%$ vs. Ia & $+24.5 \%$ vs. Ib & $+1.4 \%$ vs. Ia & $-24.9 \%$ vs. IIa & $+34.9 \%$ vs. IIb \\
\hline $\mathrm{F}$ & \multicolumn{6}{|c|}{25.72} \\
\hline$p$ & \multicolumn{6}{|c|}{$<0.001 *$} \\
\hline VC (Ia) & \multicolumn{3}{|c|}{ Virgin control group. } & \multicolumn{3}{|c|}{ *: Denotes statistical significance $(p<0.05)$. } \\
\hline $\begin{array}{l}\text { VN (Ib) } \\
\text { VN/Larg (Ic) }\end{array}$ & \multirow{2}{*}{\multicolumn{3}{|c|}{$\begin{array}{l}\text { Virgin nicotıne group. } \\
\text { Virgin nicotine and L-arginine group. }\end{array}$}} & \multirow{2}{*}{\multicolumn{3}{|c|}{$\begin{array}{l}\text { a: Denotes } p<0.05 \text { vs. VC group. } \\
\text { b: Denotes } p<0.05 \text { vs. VN group. }\end{array}$}} \\
\hline $\begin{array}{l}\text { VN/Larg (Ic) } \\
\text { PC (IIa) }\end{array}$ & & & & & & \\
\hline $\begin{array}{l}\text { PC (IIa) } \\
\text { PN (IIb) }\end{array}$ & \multicolumn{3}{|c|}{$\begin{array}{l}\text { Pregnant control group. } \\
\text { Pregnant nicotine group. }\end{array}$} & \multicolumn{3}{|c|}{ c: Denotes $p<0.05$ vs. PC group. } \\
\hline PN/Larg (IIc) & \multicolumn{6}{|c|}{$\begin{array}{l}\text { Pregnant nicotine group. } \\
\text { Pregnant nicotine and L-arginine group. }\end{array}$} \\
\hline
\end{tabular}

Daily combined nicotine exposure for four weeks induced a significant increase in renal Malondialdehyde (MDA) level, by about $78.4 \%$ and a non-significant increase in renal MDA level, by about $47.2 \%$. Addition of $0.25 \% \mathrm{~L}$-arginine to water revealed a significant decrease in the latter value of $\mathrm{VN}$ and a non-significant decrease in value of PN groups, by about $44.6 \%$ and $31.25 \%$, in comparison to VN/Larg and PN/Larg groups respectively Figs. $(3,4)$.

Daily subcutaneous injection of nicotine bitartrate, together with application of transdermal nicotine patches for four weeks induced a signifi- 
cant decrease in renal GSH level, by about $24.5 \%$ and $26.4 \%$. Addition of $0.25 \% \mathrm{~L}$-arginine to water taken by oral gavage throughout the experimental period revealed a significant increase in the latter

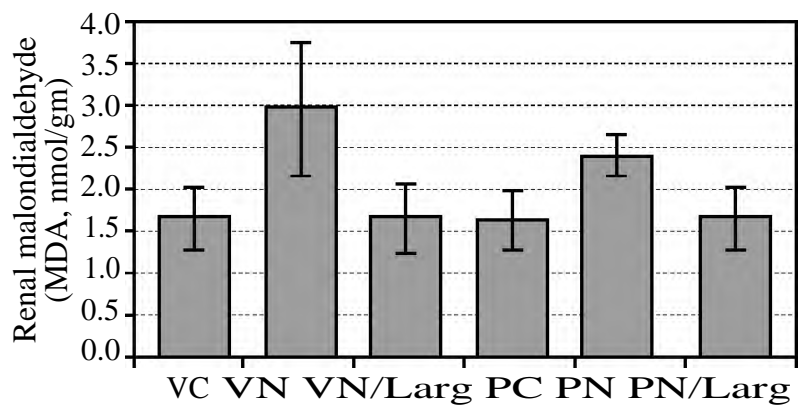

Fig. (3): Renal malondialdehyde level in virgin and pregnant rats treated with nicotine alone or combined with $\mathrm{L}$ arginine.

$\begin{array}{ll}\text { VC (Ia) } & \text { : Virgin control group. } \\ \text { VN (Ib) } & \text { : Virgin nicotine group. } \\ \text { VN/Larg (Ic) } & \text { : Virgin nicotine and L-arginine group. } \\ \text { PC (IIa) } & \text { : Pregnant control group. } \\ \text { PN (IIb) } & \text { : Pregnant nicotine group. } \\ \text { PN/Larg (IIc) } & \text { : Pregnant nicotine and L-arginine group. } \\ \text { a } & : \text { Denotes } p<0.05 \text { vs. VC group. } \\ \text { b } & : \text { Denotes } p<0.05 \text { vs. VN group. }\end{array}$

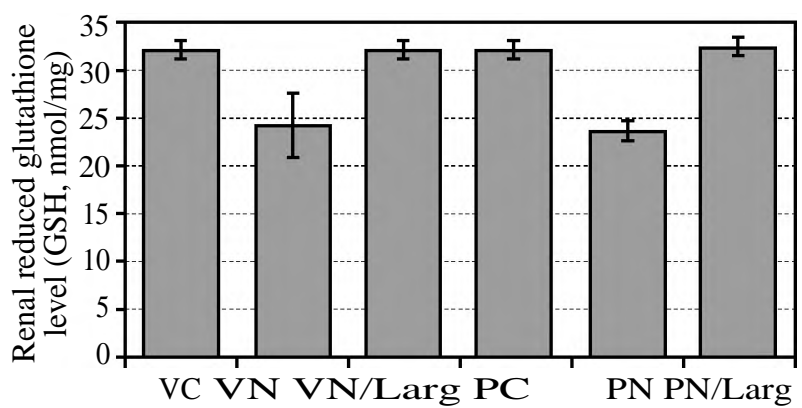

Fig. (5): Renal reduced glutathione levelin virgin and pregnant rats treated with nicotine alone or combined with Larginine.

$\begin{array}{ll}\text { VC (Ia) } & \text { : Virgin control group. } \\ \text { VN (Ib) } & \text { : Virgin nicotine group. } \\ \text { VN/Larg (Ic) } & \text { : Virgin nicotine and L-arginine group. } \\ \text { PC (IIa) } & \text { : Pregnant control group. } \\ \text { PN (IIb) } & \text { : Pregnant nicotine group. } \\ \text { PN/Larg (IIc) } & \text { : Pregnant nicotine and L-arginine group. } \\ \text { a } & : \text { Denotes } p<0.05 \text { vs. VC group. } \\ \text { b } & \text { : Denotes } p<0.05 \text { vs. VN group. } \\ \text { c } & : \text { Denotes } p<0.05 \text { vs. PC group. } \\ \text { d } & : \text { Denotes } p<0.05 \text { vs. PN group. }\end{array}$

\section{Discussion}

A major finding of the present study was that despite differences between rat and human pregnancies, changes that occur in the renal hemodynamic of both species are remarkably similar. As did humans, [4] so did pregnant rats in our experiment, that attained a significant rise in GFR, pronounced by mid-gestation when compared with virgin controls, and the subsequently associated values, of $\mathrm{VN}$ and $\mathrm{PN}$ groups, by about $32.4 \%$ and $36.7 \%$ in comparison to VN/Larg group increase $(p<0.05)$ of about $0.52 \%$ in PN/Larg group compared to PC group Figs. $(5,6)$.

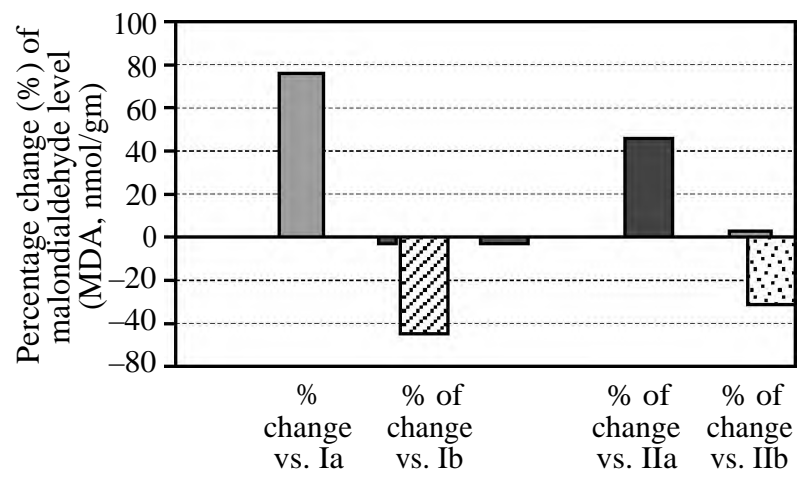

Fig. (4): Percentage change (\%) of renal malondialdehyde level in all studied groups.

VC (Ia): Virgin control group.

VN (Ib): Virgin nicotine group.

VN/Larg (Ic): Virgin nicotine and L-arginine group.

PC (IIa): Pregnant control group.

PN (IIb): Pregnant nicotine group

PN/Larg (IIc): Pregnant nicotine and L-arginine group.

a: Denotes $p<0.05$ vs. VC group.

b: Denotes $p<0.05$ vs. VN group.

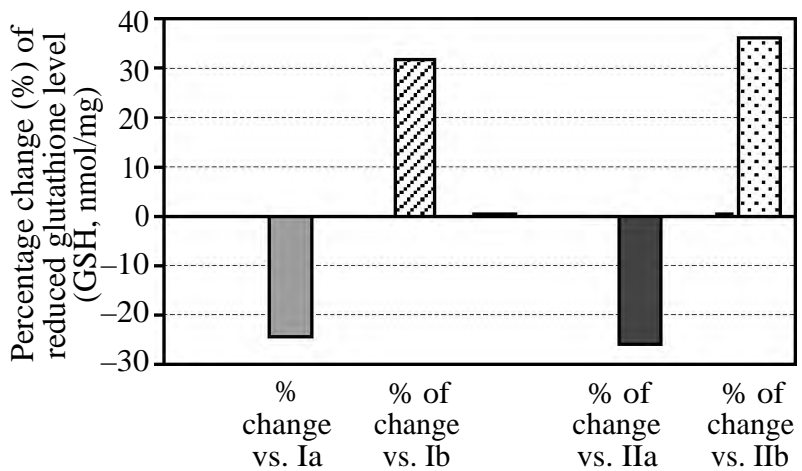

Fig. (6): Percentage change (\%) of renal reduced glutathione level in all studied groups.

$\begin{array}{ll}\text { VC (Ia) } & \text { : Virgin control group. } \\ \text { VN (Ib) } & \text { : Virgin nicotine group. } \\ \text { VN/Larg (Ic) } & \text { : Virgin nicotine and L-arginine group. } \\ \text { PC (IIa) } & \text { : Pregnant control group. } \\ \text { PN (IIb) } & \text { : Pregnant nicotine group. } \\ \text { PN/Larg (IIc) } & \text { : Pregnant nicotine and L-arginine group. } \\ \text { a } & \text { : Denotes } p<0.05 \text { vs. VC group. } \\ \text { b } & : \text { Denotes } p<0.05 \text { vs. VN group. } \\ \text { c } & : \text { Denotes } p<0.05 \text { vs. PC group. } \\ \text { d } & : \text { Denotes } p<0.05 \text { vs. PN group. }\end{array}$

with impressive rise in UFR, reflecting the typical maternal renal response to pregnancy.

These results regarding renal functional parameters in gravid group were in agreement with Davison and Noble, [5] who demonstrated that creatinine clearance increases during the luteal phase of the normal menstrual cycle and that increases in GFR during pregnancy occurred as a continuation of these changes. Paramount evidence pointed that 
renal vasodilation underlies the massive increase in Renal Plasma Flow (RPF), consequently in GFR.

Whatever the dilatory mechanisms of pregnancy, it must be potent, to ensure the reduced opposition to blood flow, as the kidneys displayed marked adaptive gestational hyperfiltration helped by profound compensatory structural and functional hypertrophy. Indeed, the insight into the mechanisms responsible for the gestational associated vasodilatory phenomena may be particularly critical, but they are not yet fully understood [2]

Authors thought that vasodilatation is accomplished mainly through increased availability of and susceptibility to the endothelium derived relaxing factor Nitric Oxide (NO) [6].

NO induces the relaxation of smooth muscles in blood vessels through guanylyl cyclase/cyclic Guanosine Monophosphate (GMP). This smooth muscle relaxation causes the dilatation of blood vessels that suppresses vascular resistance and optimizes tissue perfusion, including that in the arterioles [7] .

Indeed the actual mechanism triggers the increased bioavailability of No in normal pregnancy appears to be mediated by increase in shear stress on the vascular endothelium, by the expanded plasma volume and increase in cardiac output, triggering vascular reactivity to self-regulate tone resulting in vasodilatation. This vasomotor response proposed to be related to calcium activated potassium channels that react by shifting to an open conformation, hyperpolarizing the endothelial cell and subsequently causing calcium to enter the cell, to activate endothelial nitric oxide synthase leading to NO synthesis. Although 170 -estradiol, and progesterone have been traditionally viewed as the uterine and systemic vasodilators of pregnancy with preserved ability to stimulate NO production, these hormones have limited capacity to vasodilate the renal circulation and increase GFR [2]

The augmentation of renal hemodynamics in pregnancy is supported by enhanced secretion of relaxin, a potent vasodilator pregnancy-related hormone that has been proposed to be a major mediator of renal physiology in normal pregnancy that revealed a strong correlation with the steady rise in relaxin throughout pregnancy to a peak towards the end of the first trimester, then decline to a plateau and essentially unaltered for the remainder of gestation [8].

The results of the present work showed that 4 weeks nicotine exposure to the virgin animals resulted in significant increase in serum and urinary $\mathrm{Na}^{+}$, associated with parallel reduction in GFR and UFR, potentiating a high risk profile of renal functions, indicated by deleterious alterations in kidney markers of oxidative stress and reduction in renal protein contents. The decrease in renal GSH and increases in MDA levels, induced by chronic nicotine administration indicated that nicotine induced tissue injury and reduction in renal functions involved free radical formation. The elevated oxidative stress markers coupled with compromised antioxidant renal ability with nicotine exposure has been previously recorded in several studies, and largely accepted as one of the common mechanisms of nicotine-induced nephrotoxicity [9].

These results were in agreement with many searches which reported that nicotine induced ROS generation in a variety of tissues, and it contributed a major proportion of the net oxidative stress imposed by cigarette smoking [10].

The deleterious effects of nicotine in renal functions observed in our study could be attributed to nicotine potentiated production of ROS. It has been reported that the increase in oxidative stress stimulates certain intracellular signals, to promote the transcription of a large number of proteins involved in inflammation, apoptosis, and fibrosis, deteriorating renal functions [11]

The nicotine associated oxidative stress produces irreversible lipid peroxidation and molecular proteins damage, [12] could explain the obvious decreased renal total protein and deterioration in the renal tubular functions, in our work.

Consistent with our results, it was found that plasma $\mathrm{Na}$ level significantly increased among smokers immediately after smoking, and this may be due to stimulation of adrenal cortex, which leads to increase of circulatory cortisol that increase $\mathrm{Na}$ retention. The reduced GFR with nicotine exposure may be explained by nicotine associated sympathetic stimulation, increased peripheral vascular and renal resistances and a related increase in blood pressure [13].

Independent of the proposed nicotine associated changes in renal hemodynamics, increased overall renal tubular $\mathrm{Na}$ reabsorption via a direct action on the renal tubule is suspected with renal sympathetic stimulation. Even in the absence of a reduction in GFR or renal blood flow, sympathetic can directly impair $\mathrm{Na}$ excretion by stimulation of $\alpha 1$ adrenoceptors, located in the basolateral membrane, 
which increases $\mathrm{Na}^{+}, \mathrm{K}+$ ATPase activity and transepithelial $\mathrm{Na}$ transport [14]

Glomerulotubular balance is essential to maintain salt homeostasis in the body, where the changes in GFR are accompanied by parallel changes in tubular reabsorption, thus maintaining constant levels of fractional reabsorption of $\mathrm{Na}$ and fluids. Although the distal segments are critical to the regulation of $\mathrm{Na}$ changes in the tubular fluid, the impact of alterations in GFR is more pronounced in the proximal tubule [15].

Taking into account the final balance between the above mentioned forces, the maintained normal FENa, in the nicotine exposed group, inspite of reduction in GFR, could be explained by decreased $\mathrm{Na}$ reabsorption in the proximal renal segments secondary to decreased filtered $\mathrm{Na}$ load in glomeruli. Restoration of the renal functions, blocking the nicotine effects, through improving GFR, UFR, observed in this work could be mediated through renal vasorelaxation mostly through release of NO. L-arginine has been reported as a potential renal vasodilator which has been shown to decrease the Renal Vascular Resistance (RVR), and was consistently associated with improvement of GFR [16]

So L-arginine treatment through enhancing NO bioavailability is expected to improve the endothelial dysfunction and the impaired relaxation that is proved to be associated with nicotine exposure. The administration of L-arginine to normal animals caused significant changes in kidney function, including a marked increase in RPF, (GFR), inducing natriuresis and diuresis, increasing renal functions, and decreasing proteinuria. Arginine is welldesigned to bind the phosphate anion, and is often found in the active centres of proteins that bind phosphorylated substrates. As arginine plays an important role in maintaining the overall charge balance of a protein, preserving proteins structure [17].

L-arginine supplementation was consistently associated reduction in the renal perivascular and tubulointerstitial macrophage infiltration, leukocyte adhesion, and the formation of inflammatory mediators, possibly through NF-kB mediated mechanisms. These functional changes are supported by massive systemic and intra renal hemodynamic adaptations, mediated largely by NO and relaxin, with well documented refractoriness to various vasoconstrictors, as mentioned earlier in our discussion [18].

It was evident from our results that nicotine administration to pregnant group, like virgin group, produced a state of renal oxidative stress indicated by the significant reduction in the GSH coupled with elevated MDA and reduced total protein levels compared to control pregnant group, that may explain at least partially, the impaired renal functions, in the form of albuminuria and increased urinary $\mathrm{Na}^{+}$. Also we can concluded that nicotine exposure antagonized the NO-mediated physiological changes suspected to occur during pregnancy, where it significantly decreased the GFR and UFR, concomitant with renal NO reduction compared to control pregnant group [8]

Indeed, nicotine exposure resulted in significant reduction in GFR $84.5 \%$ in the pregnant animals versus $20.9 \%$ in virgin rats, what gave an impression that nicotine had greater effect on GFR, nearly blocked the normal pregnancy-induced glomerular hyper filtration. So the elevated expression of renal CYP1A1, in pregnant control group, explain in part the functional and hemodynamic adaptations, through enhancing formation of vasodilators, namely NO and arachidonic acid derivatives. While the down regulated expression of renal CYP1A1, with nicotine exposure, could explain the partially the deteriorated glomerular and tubular functions, through dual actions, compromised vasodilator response together with hindered nicotine metabolism [19]

Increased NO production in the kidney during pregnancy has been attributed to an up regulation of NOS, that was proved to be significantly decreased by nicotine exposure in both virgin and pregnant rats. So independently of gestation, nicotine compromised renal NO synthesis. However considering the relevance of NO to maternal adaptation to pregnancy this effect may be worse during pregnancy. The pregnancy-associated vasodilation, glomerular hyperflow, and hyperfiltration are substantially determined by relaxin, through its action on specific relaxin receptors (LGR7), that were highly expressed in the apical membranes of the proximal tubules, besides their wide distribution in blood vessel [20].

\section{Conclusion:}

This study demonstrated that chronic nicotine exposure caused more deleterious effects to pregnant rats than it did to virgin rats. Furthermore, these effects can potentially impair the adaptation of the maternal animal to pregnancy.

L-arginine treatment improved the endothelial dysfunction and the impaired relaxation that is proved to be associated with nicotine exposure. 
Administration of L-arginine to normal animals caused significant changes in kidney function.

\section{References}

1- SOMA-PILLAY P., CATHERINE N-P., TOLPPANEN H., MEBAZAA A., TOLPPANEN H. and MEBAZAA A.: Physiological changes in pregnancy. Cardiovasc. J. Afr., 27 (2): 89, 2016.

2- CONRAD K.P. and DAVISON J.M.: The renal circulation in normal pregnancy and preeclampsia: Is there a place for relaxin? Am. J. Physiol-Renal., 306 (10): F1121-F35, 2014.

3- TOMÉ L.A., YU L., De CASTRO I., CAMPOS S.B. and SEGURO A.C.: Beneficial and harmful effects of Larginine on renal ischaemia. Nephrol. Dial. Transpl.: Official publication of the European Dialysis and Transplant Association-European Renal Association, 14 (5): 1139-45, 1999.

4- LEE H.: Cystatin $\mathrm{C}$ in pregnant women is not a simple kidney filtration marker. Kid. Res. Clin. Practice, 37 (4): 313,2018

5- DAVISON J. and NOBLE M.: Serial changes in 24 hour creatinine clearance during normal menstrual cycles and the first trimester of pregnancy. BJOG: An International Journal of Obstetrics \& Gynaecology, 88 (1): 10-7, 1981.

6- LOPES VAN BALEN V., VAN GANSEWINKEL T.A. DE HAAS S., VAN KUIJK S.M., VAN DRONGELEN J., GHOSSEIN-DOHA C., et al.: Physiological adaptation of endothelial function to pregnancy: Systematic review and meta-analysis. Ultrasound. Obst. Gyn., 50 (6): 697 708, 2017.

7- PURNAMAYANTI N.M.D., WINDU S.C. and POERANTO S.: Effect of Nigella sativa Ethanol Extract on the Nitric Oxide Content and Renal Arteriole Diameter of a Pre-eclampsia Mouse Model. Eurasian. J. Med., 50 (3): $148,2018$.

8- LAFAYETTE R., HLADUNEWICH M., DERBY G., BLOUCH K., DRUZIN M. and MYERS B.: Serum relaxin levels and kidney function in late pregnancy with or without preeclampsia. Clin. Nephrol., 75 (3): 226-32, 2011.

9- JAIN A., AGRAWAL S. and FLORA S.J.: Arsenic and nicotine co-exposure lead to some synergistic effects on oxidative stress and apoptotic markers in young rat blood, liver, kidneys and brain. Toxicol. Rep., 2: 1334-46, 2015.
10-ZHOU X., SHENG Y., YANG R. and KONG X.: Nicotine promotes cardiomyocyte apoptosis via oxidative stress and altered apoptosis-related gene expression. Cardiol., 115 (4): 243-50, 2010.

11- RYAN K.M., ERNST M.K., RICE N.R. and VOUSDEN K.H.: Role of NF-icB in p53-mediated programmed cell death. Nature, 404 (6780): 892, 2000.

12- VASSALLO R., KROENING P.R., PARAMBIL J. and KITA H.: Nicotine and oxidative cigarette smoke constituents induce immune-modulatory and pro-inflammatory dendritic cell responses. Mol. Immunol, 45 (12): 33219, 2008.

13- OSMAN E., DAFALLAH A., OMER W. and ELIMIRI G.: Effect of cigarette smoking on blood sodium and potassium levels in Sudanese subjects. Int. J. Pharma. Bio. Sci., 2: B75-9, 2011.

14- SCHILLER A.M., PELLEGRINO P.R. and ZUCKER I.H.: Renal nerves dynamically regulate renal blood flow in conscious, healthy rabbits. Am. J. Physiol-Reg. I., 310 (2): R156-R66, 2015.

15- WANG T., WEINBAUM S. and WEINSTEIN A.M.: Regulation of glomerulotubular balance: Flow-activated proximal tubule function. Pflug. Arch. Eur. J. Phy., 469 (5-6): 643-54, 2017.

16- SENBEL A.M., OMAR A.G., ABDEL-MONEIM L.M., MOHAMED H.F. and DAABEES T.T.: Evaluation of 1arginine on kidney function and vascular reactivity following ischemic injury in rats: Protective effects and potential interactions. Pharmacol. Rep., 66 (6): 976-83, 2014.

17-BASHAN I., BASHAN P., SEÇILMIS M.A. and SINGIRIK E.: Protective effect of $\mathrm{L}$-arginine on gentamicin-induced nephrotoxicity in rats. Indian. J. Pharmacol, 46 (6): 608, 2014.

18- ABDELHALIM M.A.K., QAID H.A., AL-MOHY Y. and AL-AYED M.S.: Effects of quercetin and arginine on the nephrotoxicity and lipid peroxidation induced by gold nanoparticles in vivo. Int. J. Nanomed, 13: 7765, 2018.

19- HUKKANEN J., JACOB III P., PENG M., DEMPSEY D. and BENOWITZ N.L.: Effect of nicotine on cytochrome P450 1A2 activity. Brit. J. Clin. Pharmaco, 72 (5): 836-8, 2011.

20- CONRAD K.P., JEYABALAN A., DANIELSON L.A., KERCHNER L.J. and NOVAK J.: Role of relaxin in maternal renal vasodilation of pregnancy. Ann. Ny. Acad. Sci., 1041 (1): 147-54, 2005. 


\section{تآثير التعرض المزمن للنيكوتين على تكلفة الأم الكلوية فى الفئران الحواهل: الدور الهحتمل لأكسيد النيتيتريك}

الهدف من البحث: دراسة آثار التعرض المزمن الليكوتين على تكيفات الأم الكلوية أثناء الحمل والآليات الكامنة المحتملة لذلك. طرق البحث: أجريت على حب من إناث الفئران البيضاء البكر البالفة، مقسمة إلى مجموعتين رئيسيتين، تنقسم كل منهم إلى بـ مجموعات

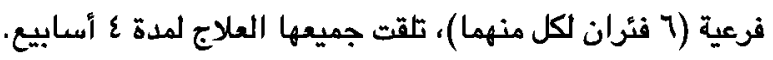

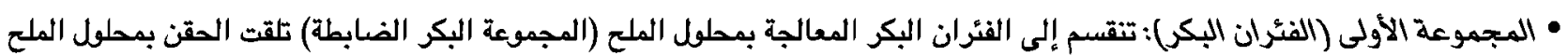

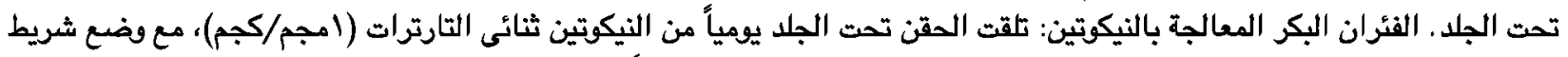

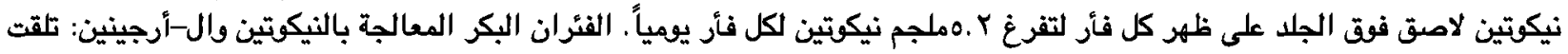

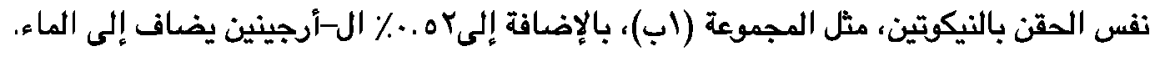

• المجموعة الثانية (الفئران الحوامل): تم تقسيمها إلى الفئران الحوامل المعالجة بمحلول الملح (المجوعة الضابطة الحوامل): تلقت الحقن

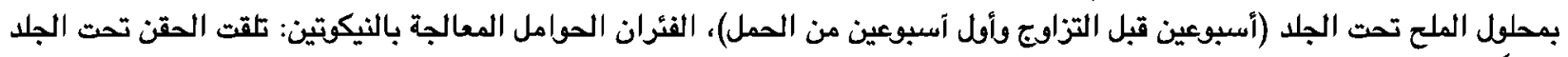

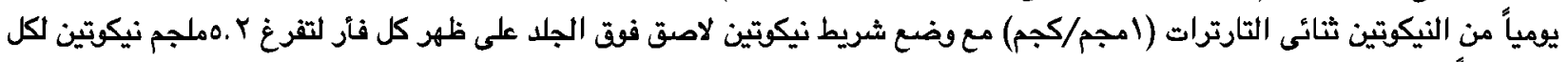

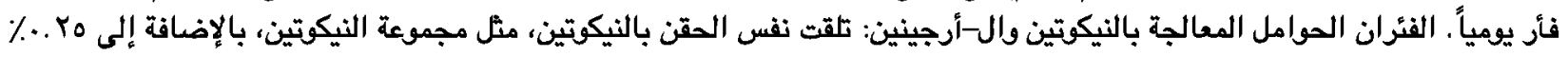

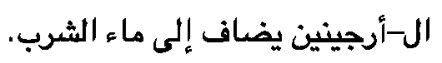

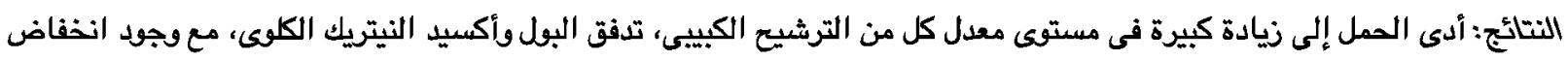

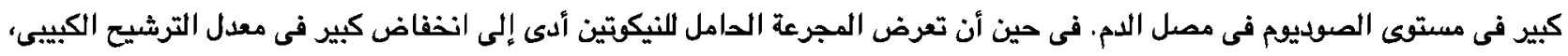

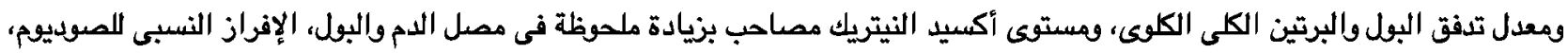

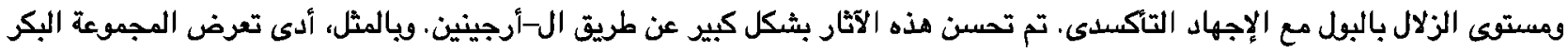

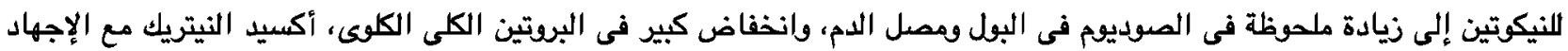

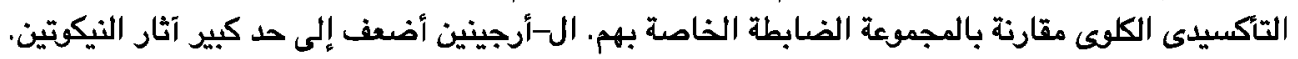

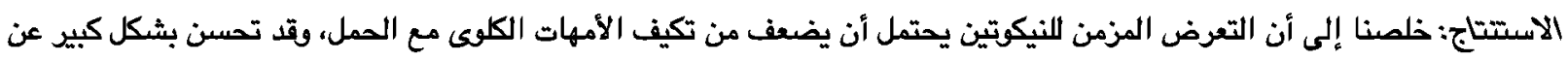

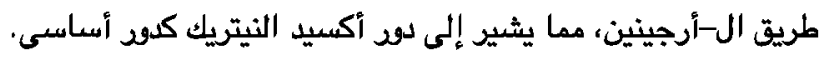

